\title{
HIPERTENSÃO APÓS TRANSPLANTE RENAL PEDIÁTRICO
}

\author{
Post-transplant pediatric kidney Hypertension
}

\begin{abstract}
Clotilde Druck Garcia', Viviane de Barros Bittencourt', Jerônimo Sperb Antonello', Filipe Dari Krüger², Magali Santos Lumertz², Vanessa Koltermann², Eduardo Pflug Comparsi², Airton Stein ${ }^{3}$, Valter Duro Garcia ${ }^{4}$.
\end{abstract}

\section{RESUMO}

Objetivo: Verificar a prevalência de hipertensão arterial sistêmica (HAS) na população pediátrica pós-transplante renal, transplantada no serviço de transplante renal do Complexo Hospitalar Santa Casa, caracterizando o grupo examinado e avaliando fatores de risco. Métodos: Os dados foram analisados em coorte retrospectiva a qual selecionou pacientes que receberam transplante renal antes dos 18 anos que realizavam acompanhamento em Ambulatório de Transplante Renal Pediátrico. Para a análise estatística, utilizou-se o coeficiente de correlação de Pearson com correção de continuidade sempre que se desejou medir o grau de correlação entre a HAS e outra variável de escala métrica. HAS foi definida com PA acima do percentil 95. Resultados: Foram analisados os dados de 150 pacientes transplantados com idade inferior a 18 anos no período de julho de 1991 a setembro de 2005 . Destes, $58 \%$ eram do sexo masculino e $89,3 \%$ brancos. A média de idade no transplante foi de 10,4 anos, e idade média atual de 15,5 anos. O tempo médio de acompanhamento foi de 57,9 meses. A prevalência de uso de corticóide foi de $86 \%$. Em relação ao esquema imunossupressor, $68,2 \%$ utilizavam tacrolimo, 27,7\%, ciclosporina e 4\%, sirolimo. A prevalência de hipertensão foi de 57,3\% nos pacientes examinados, não havendo diferença entre gênero, raça, tipo de doador (vivo ou falecido), função do enxerto ou tempo de transplante. No grupo que usava tacrolimo, a prevalência de HAS foi de 52,5\% e no que usava ciclosporina foi de $63,4 \%$, diferença não significativa $(\mathrm{p}=0,1)$. A prevalência de hipertensão entre pacientes em uso de corticosteróide foi $62,8 \%$ contra $23,8 \%$ entre os que não utilizavam $(\mathrm{p}=0,002)$. Conclusão: A ocorrência de HAS pós-transplante renal é comum. O uso de corticóide é fator de risco. Não foi identificado outro fator de risco para desenvolvimento de hipertensão.

Descritores: Transplante Renal, Hipertensão, pediatria, Imunossupressores, corticosteróides.

\footnotetext{
Instituições:

1 Serviço de Nefrologia Pediátrica do Hospital da Criança Santo Antônio, Porto Alegre

2 Acadêmicos de medicina da Universidade Federal de Ciências Saúde de Porto Alegre (UFCSPA).

${ }^{3}$ Departamento de Medicina Preventiva da Universidade Federal de Ciências da Saúde de Porto Alegre (UFCSPA).

${ }^{4}$ Serviço de Transplante Renal, Complexo Hospitalar Santa Casa, Porto Alegre
}

\section{Correspondência:}

\section{Clotilde Druck Garcia}

Rua Correa Lima, 1493 - CEP 90850-250 - Porto Alegre/RS

Fax: 5132339941

E-mail: cdgarcia1@uol.com.br

Recebido em: 30.08 .2007

Aceito em: 28.09.2007

\section{INTRODUÇÃO}

Hipertensão arterial sistêmica (HAS) é uma complicação relativamente freqüente em pacientes transplantados renais, com importantes conseqüências tanto para a sobrevida do enxerto quanto para a do paciente. ${ }^{1-2}$ Sua prevalência entre crianças transplantadas renais varia entre $50 \%$ e $80 \% .^{3-4}$ Assim como em adultos, o aumento da pressão arterial (PA) na população pediátrica transplantada renal está associado à falência do enxerto. ${ }^{5-6}$

As causas de HAS pós-transplante são multifatoriais, incluindo rejeição do enxerto, terapia imunossupressora e distúrbios vasculares renais..$^{2-7} \mathrm{O}$ controle agressivo da hipertensão nessa situação é essencial para proteger a função do enxerto a curto e em longo prazo, bem como para prevenir o desenvolvimento de doença cardiovascular (DCV) sintomática. ${ }^{4-6}$ Os eventos cardiovasculares estão entre as principais causas de morbimortalidade em longo prazo entre crianças transplantadas renais, sendo HAS e hipertrofia ventricular esquerda os eventos mais comumente observados. ${ }^{8}$

A sobrevida do enxerto renal está relacionada a diversos fatores, como: idade do paciente no transplante, tipo de doador, episódios de rejeição aguda, doença básica, tempo de isquemia, acidose tubular renal e presença de hipertensão. ${ }^{5,6,9-12} \mathrm{~A}$ diminuição da taxa de filtração glomerular também associa-se à obesidade pré-transplante e ao aumento do índice de massa corporal (IMC) na população 
pediátrica dentro do primeiro ano pós-transplante renal. Essa constatação pode dever-se ao desenvolvimento ou à permanência de hipertensão pós-transplante em pacientes severamente obesos. ${ }^{13-14}$

Transplante renal em crianças requer tradicionalmente imunossupressão com múltiplos medicamentos, incluindo corticóides. ${ }^{15} \mathrm{Os}$ agentes imunossupressores estão associados a efeitos adversos como hiperlipidemia, nefrotoxicidade e, principalmente, HAS. ${ }^{16-18}$ A função renal em pacientes tratados com ciclosporina é significativamente mais baixa do que naqueles pacientes recebendo azatioprina, embora não haja perda progressiva ao longo dos anos. ${ }^{19}$ Também verifica-se associação entre o uso de ciclosporina e a necessidade de medicamentos anti-hipertensivos. ${ }^{9}$ Ou, ainda, o manejo imunossupressor com tacrolimo requer menos medicações antihipertensivas, independentemente do uso de esteróide. ${ }^{20}$

\section{Objetivo primário:}

Verificar a prevalência de HAS pós-transplante renal em pacientes pediátricos acompanhados em ambulatorial.

\section{Objetivos secundários:}

Verificar a prevalência de HAS em relação ao esquema imunossupressor (ciclosporina ou tacrolimo); ao uso de corticóides e à função renal estimada pós-transplante.

\section{MÉTODOS}

\section{Pacientes}

Foram selecionados pacientes que receberam transplante renal antes dos 18 anos de idade e que realizavam acompanhamento em Ambulatório de Transplante Renal Pediátrico. Os critérios de exclusão foram: realização do transplante há menos de quatro meses e perda de acompanhamento ambulatorial.

Os dados foram analisados em uma coorte retrospectiva em relação a dados demográficos, presença de HAS, uso de medicamentos anti-hipertensivos e esquema de imunossupressão. Os dados demográficos considerados compreendiam idade atual, idade no transplante, número de transplantes, etnia, tempo de transplante, doença de base, IMC e taxa de filtração glomerular (TFG) estimada. Conforme esquema imunossupressor, os pacientes foram alocados entre: 1) uso ou não de corticóide (prednisona); 2) uso de ciclosporina ou tacrolimo.

HAS foi definida segundo critérios pré-estabelecidos, ${ }^{21}$ os quais a indicam como medida em três ou mais ocasiões, PA sistólica e/ou diastólica maior ou igual ao percentil 95 em relação a sexo, idade e altura. Pacientes com PA entre os percentis 90 e 95 foram considerados pré-hipertensos, e aqueles com PA inferior ao percentil 90, normotensos.

Os participantes foram classificados como possuindo "sobrepeso" ou "obesidade" quando seu IMC ultrapassava o percentil estabelecido para sua idade. ${ }^{22}$

A função renal foi estimada pela avaliação da FG, estimada através da fórmula de Schwartz, adequada para crianças e adolescentes. ${ }^{23}$ Baseado nesses cálculos, estabeleceram-se os estágios referentes à doença renal crônica dos pacientes, conforme orientação da National Kidney Foundation's. ${ }^{24}$

\section{Análise estatística}

Inicialmente, foi realizada análise descritiva dos dados demográficos, medidas de tendência central e de dispersão. Aplicou-se o teste do qui-quadrado $\left(x^{2}\right)$ para avaliar a significância das associações entre variáveis categóricas, e, para variáveis contínuas, foi utilizado o t-student. Estimou-se a magnitude das associações através do uso do coeficiente de correlação de Pearson, com correção de continuidade e intervalo de confiança (IC) de 95\%. Considerou-se nível de significância de $1 \%$. O programa estatístico utilizado foi o SPSS versão 12.0.

\section{RESULTADOS}

Analisaram-se dados de 150 pacientes acompanhados em ambulatório pediátrico de transplante renal. Entre estes, 58\% pertenciam ao sexo masculino, $89,3 \%$ eram brancos, $67,3 \%$ receberam enxerto de doador vivo e $93,3 \%$ estavam no primeiro transplante. As uropatias foram as principais doenças de base (44\%), seguidas pelas glomerulonefrites. A média de idade dos pacientes na época do transplante foi de dez anos, e o tempo médio de acompanhamento desde então, foi de aproximadamente 60 meses. A FG estimada na última consulta foi de $66,8 \mathrm{~mL} / \mathrm{min}$ e a creatinina média, de $1,47 \mathrm{mg} / \mathrm{mL}$ (tabela 1 ).

Tabela 1. Dados demográficos dos pacientes.

\begin{tabular}{|c|c|}
\hline \multicolumn{2}{|l|}{ Sexo } \\
\hline Masculino & $87(58 \%)$ \\
\hline Feminino & $63(42 \%)$ \\
\hline \multicolumn{2}{|l|}{ Doador } \\
\hline Vivo & $101(67,3 \%)$ \\
\hline Falecido & $49(32,7 \%)$ \\
\hline \multicolumn{2}{|l|}{ Número de transplantes } \\
\hline 1 & $140(93,3 \%)$ \\
\hline 2 & $9(6,0 \%)$ \\
\hline 3 & $1(0,7 \%)$ \\
\hline \multicolumn{2}{|l|}{ Etnia } \\
\hline Brancos & $134(89,3 \%)$ \\
\hline Negros & $16(10,7 \%)$ \\
\hline \multicolumn{2}{|l|}{ Doença de base } \\
\hline Uropatia & $66(44 \%)$ \\
\hline Glomerulonefrites & $35(23,3 \%)$ \\
\hline Doença hereditária & $21(14 \%)$ \\
\hline Síndrome hemolítico-urêmica & $8(5,3 \%)$ \\
\hline Doença auto-imune & $4(2,7 \%)$ \\
\hline Outras & $16(10,6 \%)$ \\
\hline Média de idade ao transplante (anos) & $10,47( \pm 4,39)$ \\
\hline Média de idade atual (anos) & $15,52( \pm 5,41)$ \\
\hline Altura média inicial (cm) & $124,09( \pm 30,02)$ \\
\hline Altura na última consulta (cm) & $144,22( \pm 22,42)$ \\
\hline Peso médio inicial (kg) & $32,89( \pm 20,61)$ \\
\hline Peso na última consulta (kg) & $45,19( \pm 16,18)$ \\
\hline IMC na última consulta (kg/m2) & $20,77( \pm 4,08)$ \\
\hline Tempo de transplante na última consulta (mês) & $57,99( \pm 44,67)$ \\
\hline Creatinina na última consulta (mg/dL) & $1,47( \pm 1,12)$ \\
\hline TFG na última consulta (mL/min) & $66,80( \pm 24,73)$ \\
\hline
\end{tabular}


Em relação ao estado nutricional dos pacientes, a maioria $(66 \%)$ foi qualificada como eutrófica, possuindo IMC médio de $20,77 \mathrm{~kg} /$ $\mathrm{m}^{2}$ na última consulta. Apenas $6 \%$ classificaram-se como obesos (segundo os parâmetros da Sociedade Brasileira de Pediatria: subnutridos - IMC nos 4 percentis mais baixos; peso médio -IMC próximo à média; acima do peso -IMC entre percentis 92 e 96, e obesos - IMC acima do percentil 96). Segundo a prevalência de HAS, 86 participantes (57\%), foram considerados hipertensos, configurando a maioria dos pacientes pediátricos que receberam transplante renal. Estes necessitaram medicação anti-hipertensiva para controle da PA. Entre eles, 41 faziam uso de apenas um antihipertensivo, 40 faziam uso de dois anti-hipertensivos e 1 fazia uso de três medicações anti-hipertensivas, os quatro restantes não faziam uso de nenhuma medicação. Além disso, os pacientes utilizavam, predominantemente, esquema imunossupressor composto por corticóides (86\%) e tacrolimo (67,3\%), como descrito na tabela 2 .

Tabela 2. Classificação dos pacientes conforme índice de massa corporal, pressão arterial pós-transplante renal e uso de corticóides e de medicamentos imunossupressores.

\begin{tabular}{ll}
\hline Estado nutricional & \\
Subnutrido & $17(11,3 \%)$ \\
Normal & $99(66,0 \%)$ \\
Sobrepeso & $25(16,7 \%)$ \\
Obesidade & $9(6,0 \%)$ \\
\hline Pressão Arterial & \\
Normotensos & $64(42,6 \%)$ \\
Hipertensos & $86(57,3 \%)$ \\
\hline Uso de corticóide & \\
Sim & $129(86,0 \%)$ \\
Não & $21(14,0 \%)$ \\
\hline Uso de imunossupressor & \\
Tacrolimo & $101(67,3 \%)$ \\
Ciclosporina & $41(27,3 \%)$ \\
Sirolimo & $6(4,0 \%)$ \\
\hline
\end{tabular}

A análise estatística verificou relação significativa $(p=0,002)$ entre o uso de corticóides e IMC. O IMC médio dos pacientes que utilizavam esse tipo de medicamento foi de $21,2 \mathrm{Kg} / \mathrm{m}^{2}$ (desvio padrão de 4,1 ) versus $18,2 \mathrm{Kg} / \mathrm{m} 2$ (desvio padrão de 2,7 ) naqueles que não o utilizaram. Além disso, dos nove pacientes classificados na amostra como obesos, oito utilizavam corticóide, e sete eram hipertensos (tabela 3).

Tabela 3. Utilização de corticóide e índice de massa corporal.

\begin{tabular}{ll}
\hline Uso de corticóide & IMC \\
Sim & $21,2 \mathrm{~kg} / \mathrm{m} 2( \pm 4,1)^{*}$ \\
Não & $18,2 \mathrm{~kg} / \mathrm{m} 2( \pm 2,7)$ \\
\hline
\end{tabular}

${ }^{*} p=0,002$
Ainda, houve associação entre HAS e uso de corticóides ( $\mathrm{p}=0,001)$. O risco relativo estimado (odds ratio) para desenvolvimento de HAS devido ao uso de corticóides após o transplante foi de 5,4 (1,8-15,6, IC $95 \%$ - tabela 4). Não se evidenciou relação estatisticamente significativa entre os diferentes esquemas imunossupressores (ciclosporina ou tacrolimo; $\mathrm{p}=0,1$ ) e a prevalência de HAS. Também não houve diferença significativa entre hipertensos e normotensos em relação à função renal estimada pela TFG estimada durante o seguimento $(\mathrm{p}=0,23)$.

Tabela 4. Características de normotensos e de hipertensos em relação à medicação imunossupressora e à taxa de filtração glomerular (TFG).

\begin{tabular}{|c|c|c|}
\hline & Normotensos & Hipertensos \\
\hline \multicolumn{3}{|c|}{ Uso de corticóide } \\
\hline Sim & $48(37,2 \%)$ & $81(62,8 \%)^{\star}$ \\
\hline Não & $16(76,2 \%)$ & $5(23,8 \%)$ \\
\hline \multicolumn{3}{|c|}{ Imunossupressor } \\
\hline Ciclosporina & $15(36,6 \%)$ & $26(63,4 \%)$ \\
\hline Tacrolimo & $48(47,5 \%)$ & $53(52,5 \%)$ \\
\hline TFG (mg/dL) & $64( \pm 22,5)$ & $69( \pm 26,2)$ \\
\hline
\end{tabular}

\section{DISCUSSÃO}

Hipertensão é uma complicação freqüente após transplante renal pediátrico, ${ }^{6-9}$ sendo sua prevalência variável entre os diversos estudos. Em nossa amostra, 57\% das crianças transplantadas renais foram classificadas como hipertensas, taxa que, além de representar a maioria das crianças submetidas a transplante renal, concorda com a maioria das informações verificadas na literatura..$^{2-4 ; 8}$

O risco de desenvolvimento de HAS pós-transplante foi relacionado, significativamente, ao uso de corticóides (risco relativo de 5,4). Atualmente, há uma ampla discussão na literatura sobre o uso de protocolos imunossupressores contendo ou não o uso de corticosteróides. ${ }^{15,25,26}$ A maioria dos estudos, inclusive de revisão, afirma haver melhora dos parâmetros de PA, porém, elevação da taxa de rejeição aguda do enxerto sem seu uso. Debate-se a possibilidade do uso de outras drogas imunossupressoras ou de um maior número destas ou redução da dose de corticosteróides, para que as taxas de rejeição aguda do enxerto sejam idênticas ou diminuídas, e para que os efeitos colaterais como HAS advindos dos corticóides sejam evitados. Estudo comparando esquema imunossupressor com e sem uso de esteróides demonstrou semelhança entre a PA sistólica dos pacientes dos dois grupos. Entretanto, os autores associaram em parte essa similaridade à necessidade de maiores quantidades de fármacos para controlar a PA pelo grupo cuja imunossupressão foi realizada com esteróides. ${ }^{15}$

O uso de corticóides também está associado a aumento do IMC após o transplante. Os pacientes faziam uso de prednisona na dose de $0,1 \mathrm{mg} / \mathrm{kg} / \mathrm{dia}$ ou $0,2 \mathrm{mg} / \mathrm{kg} / \mathrm{em}$ dias alternados. Observa-se crescente incidência de obesidade nesse grupo de pacientes (presente em $6 \%$ da nossa amostra), condição que tende a elevar o número de complicações pós-transplante, especialmente em algumas faixas etárias, como dos 6 aos 12 anos, na qual acredita-se haver maior risco de morte, principalmente por doenças cardio-pulmonares. ${ }^{27}$ 
Além disso, a obesidade prévia ao transplante está associada a menor taxa de filtração glomerular após enxerto renal, fato que pode decorrer da persistência ou do surgimento de HAS póstransplante em pacientes severamente obesos. ${ }^{14}$ Em adultos, há forte evidência de que há associação entre obesidade e complicações pós-transplante, independente de outros fatores de risco a estas. ${ }^{28}$ Entretanto, isso ainda não pode ser afirmado em relação à população pediátrica pela necessidade de mais estudos.

Durante o período de acompanhamento ambulatorial, não se encontrou relação entre aumento da PA e os diferentes esquemas imunossupressores, compostos por ciclosporina ou tacrolimo. Por outro lado, estudos demonstraram haver associação entre maior necessidade de controle da PA e uso de ciclosporina, se comparado ao regime com tacrolimo..$^{9-20-29}$ Amplo estudo demonstrou que pacientes em uso de tacrolimo apresentavam menor necessidade de uso de anti-hipertensivos e, também, melhor função renal após dois anos de transplante. ${ }^{30}$

Não houve diferença significativa entre normotensos e hipertensos em relação à função renal estimada pós-transplante em aproximadamente cinco anos de acompanhamento $(p=0,23)$. Outros estudos mostram que a ocorrência de HAS pós-transplante resulta em piora da função do enxerto e em aumento da dosagem média de creatinina. ${ }^{5-6}$ Contudo, a sobrevida média de pacientes hipertensos que possuíam controle adequado da PA foi semelhante a de indivíduos normotensos em um período de cinco anos. ${ }^{3}$ Em uma ampla amostra, não foi encontrada relação entre HAS e disfunção do enxerto renal, mas sim associação entre a necessidade de anti-hipertensivos e piora funcional deste. ${ }^{9}$

\section{CONCLUSÃO}

A ocorrência de HAS pós-transplante renal é comum. Em crianças transplantadas renais, o uso de corticóide é fator de risco para desenvolvimento de HAS. Este estudo não identificou correlação entre esquema imunossupressor e HAS, nem diferença entre normotensos e hipertensos em relação à função renal estimada póstransplante ou qualquer outro fator de risco para desenvolvimento de HAS nessa população de pacientes.

\section{ABSTRACT}

Purpose: To determine the prevalence of post-renal transplantation hypertension in a pediatric renal transplant population at Complexo Hospitalar Santa Casa, characterizing the group and verifying associated risk factors. Patients and methods: Data was analyzed in a retrospective cohort of pediatric renal transplant recipients who were grafted before the age of 18. For statistical analysis, the Pearson correlation coefficient was used, with correction of continuity. Hypertension was assumed if BP was above the 95 percentile. Results: 150 patients transplanted for the period between July 1991 and September, 2005 were enrolled. 58\% were males. Mean age at transplantation was 10,4 years, and patients had a mean age of 15.5 years at the time of the data collection. There was a prevalence of Caucasians ( $89.3 \%)$. Mean follow-up time was 57.9 months. Immunosuppressant therapy included tacrolimus (68.2\%), cyclosporine (27.7\%), and sirolimus (4\%) with no calcineurin inhibitors. Steroids were used in $86 \%$ of patients. Hypertension was present in $86(57.3 \%)$ patients. There was no significant difference in the prevalence of hypertension related to gender, race, donor (living or deceased), graft function, and follow-up time. HTN was present in $52.5 \%$ patients receiving tacrolimus, $63.4 \%$ in those receiving cyclosporine ( $p=0,1)$. In the group on steroids, $62.8 \%$ were hypertensive versus $23.8 \%$ in the steroid-free group $(\mathrm{p}=0.002)$. Conclusion: Hypertension is prevalent after renal transplantation. The use of steroids in kidney transplant recipients is a risk factor to develop hypertension. No other risk factor for high blood pressure was identified.

Keywords: kidney transplantation, hypertension, pediatrics, immunosuppressive agents, corticosteroids

\section{REFERÊNCIAS}

1. Berber I, Aydin C, Yigit B, Kara VM, Yildar M, Duzyol C, et al. Blood pressure and graft outcome in renal transplantation. Transplant Proc. 2005;37:997-8.

2. Baluarte HJ, Gruskin AB, Ingelfinger JR, Stablein D, Tejani A. Analysis of hypertension in children post renal transplantation--a report of the North American Pediatric Renal Transplant Cooperative Study (NAPRTCS). Pediatr Nephrol. 1994;8:570-3.

3. Kim HC, Kwon JK, Park SB, Cho WH, Park CH. Hypertension in kidney transplantation recipients: effect on long-term renal allograft survival. Transplant Proc. 1998;30:3906-7.

4. Mitsnefes MM. Hypertension and end-organ damage in pediatric renal transplantation. Pediatr Transplant. 2004;8:394-9.

5. Mitsnefes MM, Khoury PR, McEnery PT. Early posttransplantation hypertension and poor long-term renal allograft survival in pediatric patients. J Pediatr. 2003;143:98-103.
6. Mitsnefes MM, Omoloja A, McEnery PT. Short-term pediatric renal transplant survival: blood pressure and allograft function. Pediatr Transplant. 2001;5:160-5.

7. Buscher R, Vester U, Wingen AM, Hoyer PF. Pathomecanisms and the diagnosis of arterial hypertension in pediatric renal allograft recipients. Pediatr Nephrol. 2004;19:1202-11.

8. Kitzmueller E, Vecsei A, Pichler J, Bohm M, Muller T, Vargha R, et al. Changes of blood pressure and left ventricular mass in pediatric renal transplantation. Pediatr Nephrol. 2004;19:1385-9.

9. Sorof JM, Goldstein SL, Brewer ED, Steiger HM, Portman RJ. Use of antihypertensive medications and post-transplant renal allograft function in children. Pediatr Transplant. 2000;4:21-7.

10. Sorof JM, Sullivan EK, Tejani A, Portman RJ. Antihypertensive medication and renal allograft failure: a North American Pediatric Renal Transplant Cooperative Study report. J Am Soc Nephrol. 1999;10:1324-30. 
11. Bartosh SM, Alonso EM, Whitington PF. Renal outcomes in pediatric liver transplantation. Clin Transplant. 1997;11:354-60.

12. El-Husseini AA, Foda MA, Shokeir AA, Shehab El-Din AB, Sobh MA, Ghoneim MA. Determinants of graft survival in pediatric and adolescent live donor kidney transplant recipient: A single center experience. Pediatr Transplant. 2005;9(6):763-9.

13. Omoloja A, Stolfi A, Mitsnefes M. Pediatric obesity at renal transplantation: A single center experience. Pediatr Transplant. 2005;9:770-2.

14. Mitsnefes MM, Khoury P, McEnery PT. Body mass index and allograft function in pediatric renal transplantation. Pediatr Nephrol. 2002;17:535-9.

15. Silverstein DM, Aviles DH, LeBlanc PM, Jung FF, Vehaskari VM. Results of one-year follow-up of steroid-free immunosuppression in pediatric renal transplant patients. Pediatr Transplant. 2005;9:589-97.

16. Ferraris JR, Ghezzi L, Waisman G, Krmar RT. Potential cardiovascular risk factors in paediatric renal transplant recipients. Pediatr Nephrol. 2006;21:119-25.

17. Trompeter R, Filler G, Webb NJ, Watson AR, Milford DV, Tyden G, et al. Randomized trial of tacrolimus versus cyclosporin microemulsion in renal transplantion. Pediatr Nephrol. 2002;17:141-9.

18. Serdaroglu E, Mir S, Berdeli A. Hypertension and ace gene insertion/deletion polymorphism in pediatric renal transplant patients. Pediatr Transplant. 2005;9:612-7.

19. Brodehl J, Bokenkamp A, Hoyer PF, Offner G. Long-term results of cyclosporin A therapy in children. J Am Soc Nephrol. 1992;2:246-54.

20. Ellis D, Shapiro R, Jordan ML, Scantlebury VP, Gilboa N, Hopp L, et al. Comparison of FK-506 and cyclosporine regimens in pediatric renal transplantation. Pediatr Nephrol. 1994;8:193-200.

21. National High Blood Pressure Education Program Working Group on High Blood Pressure in Children and Adolescents. The fourth report on the diagnosis, evaluation, and treatment of high blood pressure in children and adolescents. Pediatrics. 2004;114:555-76.
22. Cole TJ, Bellizzi MC, Flegal KM, Dietz WH. Establishing a standard definition for child overweight and obesity worldwide: international survey. BMJ. 2000;320:1240-3.

23. Schwartz GJ, Brion LP, Spitzer A. The use of plasma creatinine concentration for estimating glomerular filtration rate in infants, children, and adolescents. Pediatr Clin North Am. 1987;34:571-90.

24. Hogg RJ, Furth S, Lemley KV, Portman R, Schwartz GJ, Coresh J, et al; National Kidney Foundation's Kidney Disease Outcomes Quality Initiative. National Kidney Foundation's Kidney Disease Outcomes Quality Initiative clinical practice guidelines for chronic kidney disease in children and adolescents: evaluation, classification, and stratification. Pediatrics. 2003;111:1416-21.

25. Vidhun JR, Sarwal MM. Corticosteroid avoidance in pediatric renal transplantation. Pediatr Nephrol. 2005;20:418-26.

26. Vidhun JR, Sarwal MM. Corticosteroid avoidance in pediatric renal transplantation: can it be achieved? Pediatr Drugs. 2004;6:273-87.

27. Hanevold CD, Ho PL, Talley L, Mitsnefes MM. Obesity and renal transplant outcome: a report of the North American Pediatric Renal Transplant Cooperative Study. Pediatrics. 2005;115:352-6.

28. el-Agroudy AE, Wafa EW, Gheith OE, Shehab el-Dein AB, Ghoneim MA. Weight gain after renal transplantation is a risk factor for patient and graft outcome. Transplantation. 2004;77:1381-5

29. Morales JM, Andres A, Rengel M, Rodicio JL. Influence of cyclosporin, tacrolimus and rapamycin on renal function and arterial hypertension after renal transplantation. Nephrol Dial Transplant. 2001;16:121-4.

30. Neu AM, Ho PL, Fine RN, Furth SL, Fivush BA. Tacrolimus vs. cyclosporine A as primary immunosuppression in pediatric renal transplantation: a NAPRTCS study. Pediatr Transplant. 2003;7:217-22. 